Measurements of interactions between waves and energetic ions in basic plasma experiments

This article has been downloaded from IOPscience. Please scroll down to see the full text article.

2012 Plasma Phys. Control. Fusion 54124007

(http://iopscience.iop.org/0741-3335/54/12/124007)

View the table of contents for this issue, or go to the journal homepage for more

Download details:

IP Address: 128.178.125.98

The article was downloaded on 26/11/2012 at 12:24

Please note that terms and conditions apply. 


\title{
Measurements of interactions between waves and energetic ions in basic plasma experiments
}

\author{
W W Heidbrink ${ }^{1}$, H Boehmer ${ }^{1}$, R McWilliams ${ }^{1}$, A Preiwisch ${ }^{1}$, Y Zhang $^{1}$, \\ L Zhao ${ }^{1,4}$, S Zhou ${ }^{1,5}$, A Bovet $^{2}$, A Fasoli ${ }^{2}$, I Furno ${ }^{2}$, K Gustafson ${ }^{2}$, \\ P Ricci ${ }^{2}$, T Carter ${ }^{3}$, D Leneman ${ }^{3}$, S K P Tripathi ${ }^{3}$ and S Vincena ${ }^{3}$ \\ ${ }^{1}$ Department of Physics and Astronomy, University of California, Irvine, CA, USA \\ ${ }^{2}$ Centre de Recherches en Physique des Plasmas, Ecole Polytechnique Federale de Lausanne, CRPP, \\ Lausanne, Switzerland \\ ${ }^{3}$ Department of Physics and Astronomy, University of California, Los Angeles, CA, USA
}

Received 2 July 2012, in final form 17 August 2012

Published 21 November 2012

Online at stacks.iop.org/PPCF/54/124007

\begin{abstract}
To measure the transport of fast ions by various types of waves, complementary experiments are conducted in linear and toroidal magnetic fields in the large plasma device and the toroidal plasma experiment. Lithium sources that are immersed in the plasma provide the energetic ions. Spatial scans of collectors measure the transport. Techniques to find the beam and optimize the spatial sensitivity are described. Measurements of Coulomb scattering, resonant interaction with Alfvén waves, and transport by drift-wave and interchange turbulence are summarized.
\end{abstract}

(Some figures may appear in colour only in the online journal)

\section{Introduction}

Energetic particles are common in both natural and laboratory plasmas. Wave-particle interactions create supra-thermal populations in many natural plasmas, including the solar corona [1] and the aurora [2]. In magnetic fusion experiments, neutral beam injection, radio frequency heating and fusion reactions produce supra-thermal ion populations [3]. In both environments, detailed diagnosis of the energetic ions and their interactions is challenging. In space, the large spatial scales and expense of satellite exploration limit progress while, in fusion plasmas, the high temperatures mandate indirect diagnostic techniques.

In the last decade, two basic plasma physics facilities have developed programs for detailed study of interactions between waves and energetic ions. One facility, the large plasma device (LAPD) at the University of California, Los Angeles [4], employs linear magnetic field geometry while the other, the toroidal plasma experiment (TORPEX) at the École Polytechnique Fédérale de Lausanne [5], has a

\footnotetext{
${ }^{4}$ Present affiliation: FAR-TECH, Inc., San Diego, CA, USA.

${ }^{5}$ Present affiliation: Intel Corporation, Hillsboro, OR, USA.
}

toroidal magnetic field. Both experiments successfully mimic important aspects of the fast-ion physics of less accessible plasmas. As in natural and fusion plasmas, the energetic ions have speeds $v_{\mathrm{f}}$ that are intermediate between the electron thermal speed $v_{\mathrm{e}}$ and the ion thermal speed $v_{\mathrm{i}}, v_{\mathrm{e}} \gg v_{\mathrm{f}} \gg$ $v_{\mathrm{i}}$. The fast-ion gyroradius $\rho_{\mathrm{f}}$ is much larger than the ion and electron gyroradii $\rho_{\mathrm{i}}$ and $\rho_{\mathrm{e}}$. The plasmas are more collisional than their natural and fusion counterparts but the timescales for wave-particle interaction are still shorter than characteristic collision times. Although the experiments cannot perfectly replicate fusion and space plasmas, they have a major advantage: because solid objects can be inserted into the plasma, detailed characterization of both the wave fields and the fast-ion population is possible.

The experiments to date have concentrated on dilute energetic ion populations, where the fast ions are effectively test particles. This paper reviews progress in the first decade of research. Experiments with an intense fast-ion source are also underway [6] but are not reported here. The paper begins with descriptions of the fast-ion source and the experimental techniques (section 2). Section 3 summarizes completed physics studies, while section 4 discusses some planned upgrades and future experiments. 

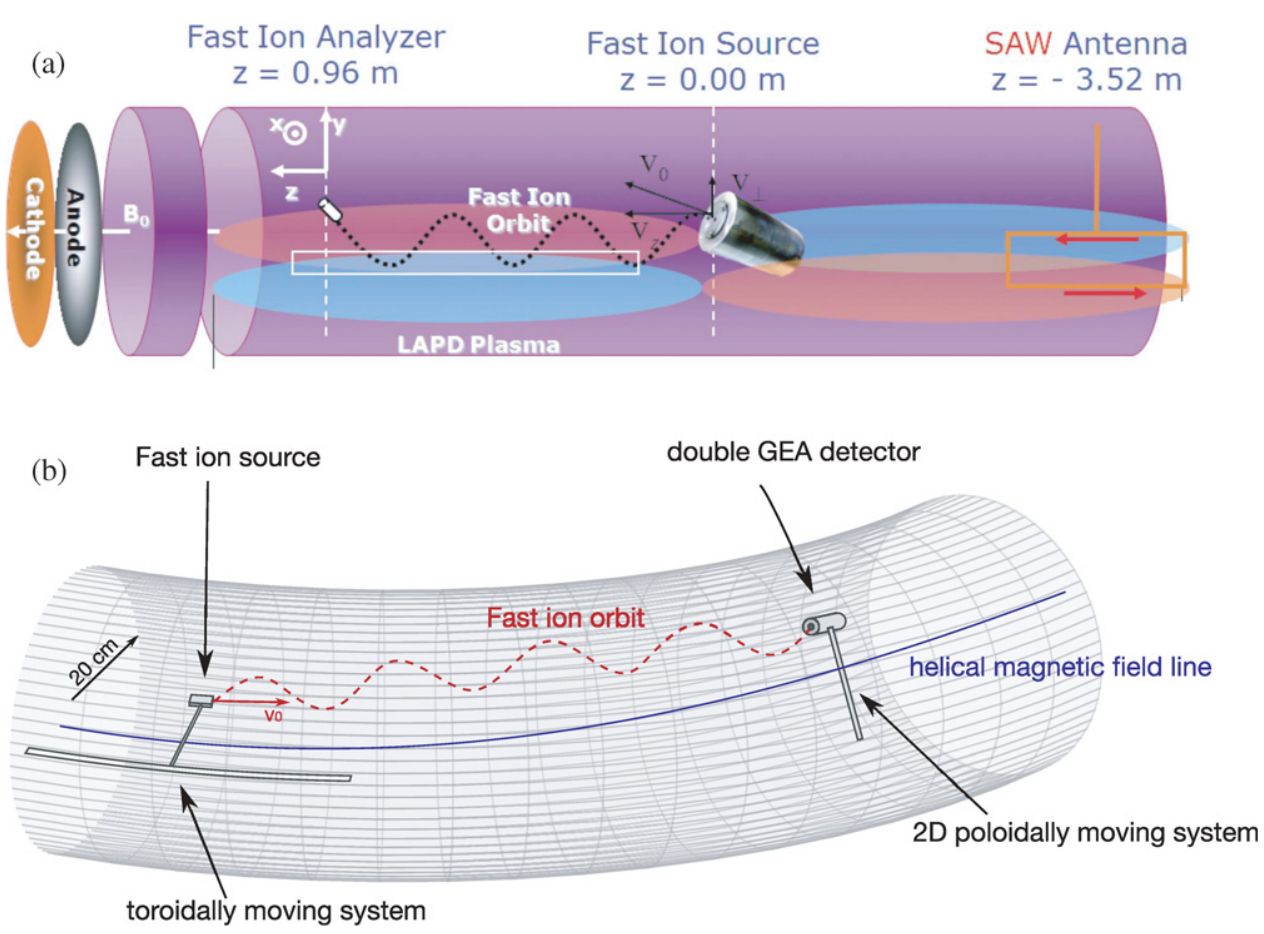

Figure 1. Illustrations of representative $(a)$ LAPD and $(b)$ TORPEX experiments. LAPD diagram reprinted with permission from [22]. Copyright 2008, American Institute of Physics.

\section{Experimental technique}

The basic idea of a typical experiment is simple (figure 1). A fast-ion source is immersed into the plasma and emits ions that orbit in the magnetic field. Meanwhile, an antenna (or the plasma itself) launches waves that interact with the fast ions. Probes and optical diagnostics characterize the wave fields, while specialized collectors measure the effect of the waves on the spatial profile of the fast-ion beam.

\subsection{Fast-ion sources}

At the LAPD, the source $[7,8]$ uses a ceramic aluminosilicate emitter that is loaded with lithium. The ceramic is heated with $\sim 80 \mathrm{~W}$ to $\sim 1000^{\circ} \mathrm{C}$. The emitter and grids are housed in a cylindrical shield (figure 2 ) that usually floats electrically. The emitter is biased positively relative to the housing to accelerate the beam. Extracted energies of $300-600 \mathrm{eV}$ with an energy spread of $\lesssim 20 \mathrm{eV}$, divergence of $\sim 3^{\circ}$, and current density of $\sim 100 \mu \mathrm{A} \mathrm{cm}^{-2}$ are typical for a properly functioning emitter. The pitch angle of the emitted beam is adjusted by rotating the source relative to the magnetic field. The source works well even for pitch angles approaching $90^{\circ}$ [9]. A calibration experiment [8] showed that $\gtrsim 94 \%$ of the emitted ions are ${ }^{7} \mathrm{Li}$.

At TORPEX, the source is based on a similar concept, but to limit the perturbations to a smaller plasma volume, and to provide a continuous toroidal reconstruction, two major innovations have been implemented. Firstly, design improvements produced increased emission in a smaller source $[10,11]$. The source uses a small $\mathrm{Li}$ ion emitter with a diameter of $6.35 \mathrm{~mm}$ and a boron-nitride casing (figure $2(b)$ ). The emitter is typically heated with $\sim 20 \mathrm{~W}$ to $\sim 1100^{\circ} \mathrm{C}$ and emits a fast ion current density of $\sim 35 \mu \mathrm{A} \mathrm{cm}^{-2}$. The energy can be
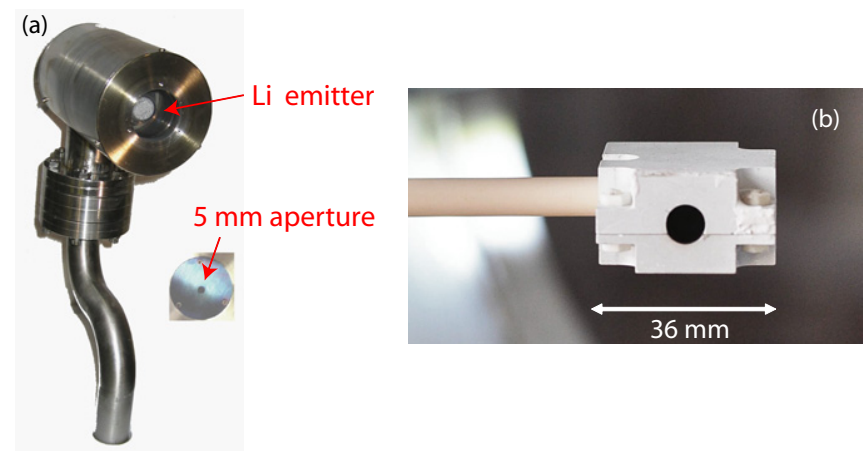

Figure 2. Photographs of the (a) LAPD and $(b)$ TORPEX lithium fast-ion sources.

changed from $\sim 50 \mathrm{eV}$ to $1 \mathrm{keV}$. Secondly, in its present setup, the source is mounted on a motorized movable system and can be continuously moved over a toroidal distance of $50 \mathrm{~cm}$.

The first experiments on the LAPD used an argon RF source [12]. The lithium sources have two important advantages: they can operate at pitch angles $>25^{\circ}$ and they produce negligible amounts of neutral gas. A disadvantage of the lithium sources is that they have a finite lifetime and, if purchased commercially, are rather expensive.

\subsection{Measuring the fast-ion beam}

Since the plasma density is much larger than the fast-ion density, detection of the fast-ion beam requires specialized collectors. The standard detector at the LAPD [8] utilizes both biased grids and collimation (figure 3(a)) to suppress interference from the background plasma. Early LAPD experiments $[13,14]$ used only biased grids in the relatively 

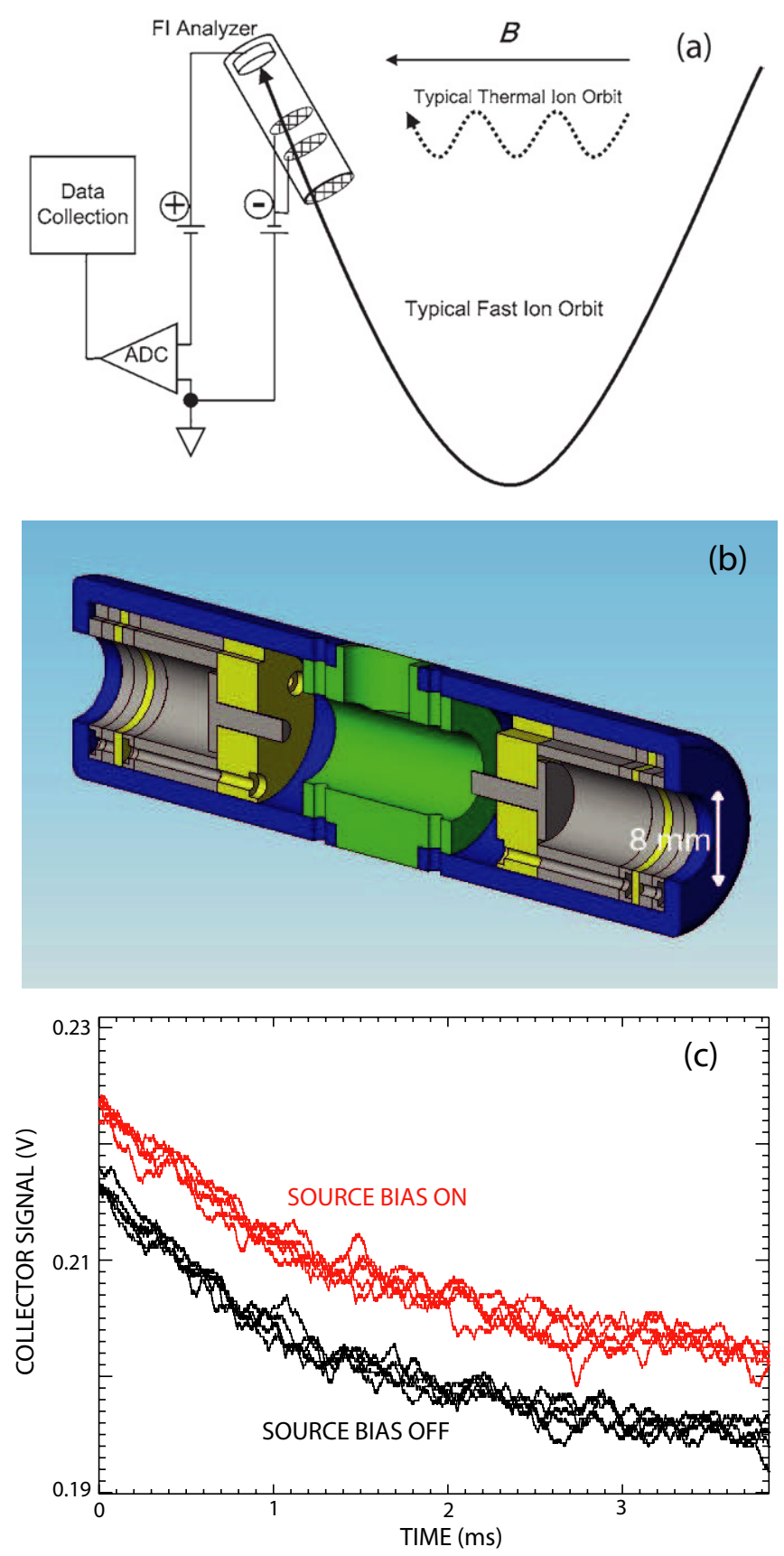

Figure 3. (a) Schematic diagram of the LAPD collector that uses a combination of collimation and biased grids to suppress thermal contributions to the signal. Reprinted with permission from [7]. Copyright 2007, American Institute of Physics. (b) Schematic of the double gridded energy analyzer used in TORPEX with a synchronous detection scheme. (c) Emitter bias and collector signals for 10 successive LAPD shots. The fast-ion current is inferred from the average difference of the signals.

tenuous 'afterglow' LAPD plasma but this collector was overwhelmed by electron currents in the denser, active-phase portion of the discharge. The standard collimated/gridded detector is unable to measure the energy of the beam (probably due to charge accumulation on insulators), so a new collector was designed and successfully tested [15].

In TORPEX, the standard detector is a double gridded energy analyzer (figure 3(b)) $[10,16]$ that utilizes differential measurement between the two collector signals to cancel plasma contributions to the signal. It is mounted on a $2 \mathrm{D}$ motorized movable system which can position it at almost any point of the poloidal cross-section (figure $1(b)$ ).

Both facilities employ averaging to improve the signalto-noise ratio. The LAPD device creates a $\sim 10$ ms duration plasma every second. In normal operation, the emitter bias is applied on alternating shots. The detected fast-ion current is the difference between 5 'beam-on' shots and 5 'beam-off' shots (figure $3(c)$ ). In TORPEX, the discharges can last up to $10 \mathrm{~min}$ while the emitter biasing voltage is modulated at $\sim 1 \mathrm{kHz}$ and the signal from the gridded energy analyzer is lock-in detected to obtain the fast-ion current. In order to remove the undesired capacitively coupled noise arising from the source bias modulation, an analog lock-in amplifier with the possibility to set a 'dead time' is used [16].

Multiple measurements of this sort yield a beam profile (figure 4). The data are collected in a spatial plane that is perpendicular to the magnetic field. Since the LAPD field is normally linear, the unperturbed fast-ion orbit is a helix. The beam spot has a simple interpretation. The measured plane is at an axial distance $z$ from the fast-ion source. The gyroangle $\varphi$ at $z$ is simply $\varphi=\Omega_{\mathrm{f}} t=\Omega_{\mathrm{f}} z / v_{z}$, where $\Omega_{\mathrm{f}}$ is the fastion gyrofrequency and $v_{z}$ is the axial velocity. Changes in the axial velocity (parallel energy) manifest themselves as shifts in gyroangle, i.e. as vertical spatial shifts for the orientation of figure 4(a). Shifts in the opposite direction (horizontal in figure $4(a)$ ) are caused by changes in fast-ion gyroradius $\rho_{\mathrm{f}}$ (perpendicular velocity) or by shifts in the guiding center position. To analyze the plane data, the unperturbed orbit is projected onto the plane and intensity-weighted profiles in the radial and gyroangle directions are inferred [7,8]. Usually, the profiles are close to Gaussian in shape and spreading of the beam is simply described by the full-width half-maximum (FWHM) of the beam spot.

To achieve maximum sensitivity to wave-induced spreading in the radial $r$ direction, the beam spot is measured at gyroangles of $2 \pi n$, where $n$ is an integer. (The collisionless peristaltic contraction and expansion of a finite divergence beam is a well-known phenomenon in the particle accelerator community.) As shown in figure 7 of [13], the radial width is $\sim 1 / 2$ as large for the optimum gyroangle. In standard practice on the LAPD, the energy, pitch angle, magnetic field and number of cyclotron orbits are chosen to satisfy $2 \pi n=\Omega_{\mathrm{f}} z / v_{z}$ at the selected axial position of the spatial plane. (As shown in figure 11(a), peristaltic oscillations in beam width are also observed at TORPEX.)

In principle, with these selections, the beam returns to the $(r, \varphi)$ of the source at the selected $z$. In practice, finding the beam can be challenging. In TORPEX, installation of a toroidal rail that accommodates 3D measurements of the fastion orbits (figure $1(b)$ ) has overcome this difficulty. At the LAPD, a standard procedure is to locate the beam initially in the quiet afterglow plasma (where the signal-to-noise on the collector signal is larger). If the beam is elusive, the beam energy is ramped down. This creates a spike in collector signal each time the beam completes a cyclotron orbit (figure 5). The beam or magnetic field parameters are then adjusted so that the beam completes an integer number of cyclotron orbits at the desired axial location. 

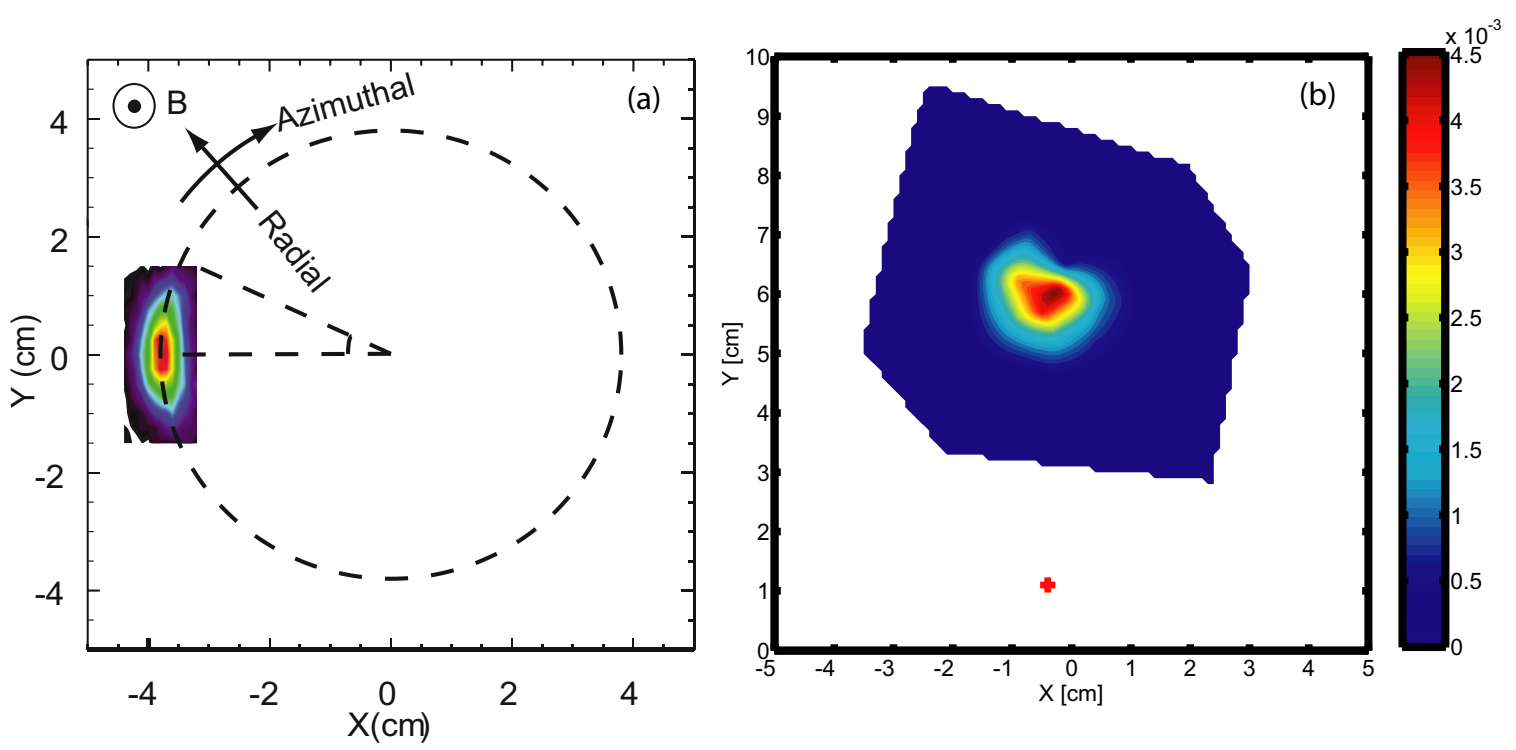

Figure 4. (a) Projection of the fast-ion orbit onto a horizontal plane in the LAPD, with the beam profile measured three ports from the source overlaid. For this orientation of the source, radial motion causes horizontal spreading, while changes in parallel velocity cause vertical spreading. Reprinted with permission from [25]. Copyright 2010, American Institute of Physics. (b) Poloidal fast ion current density profile (a.u.) in TORPEX, for ions of $70 \mathrm{eV}$ at a toroidal distance of $54 \mathrm{~cm}$ from the source. The red cross indicates the source position. The beam spot is shifted upward because of the $\nabla B$ and curvature drifts.

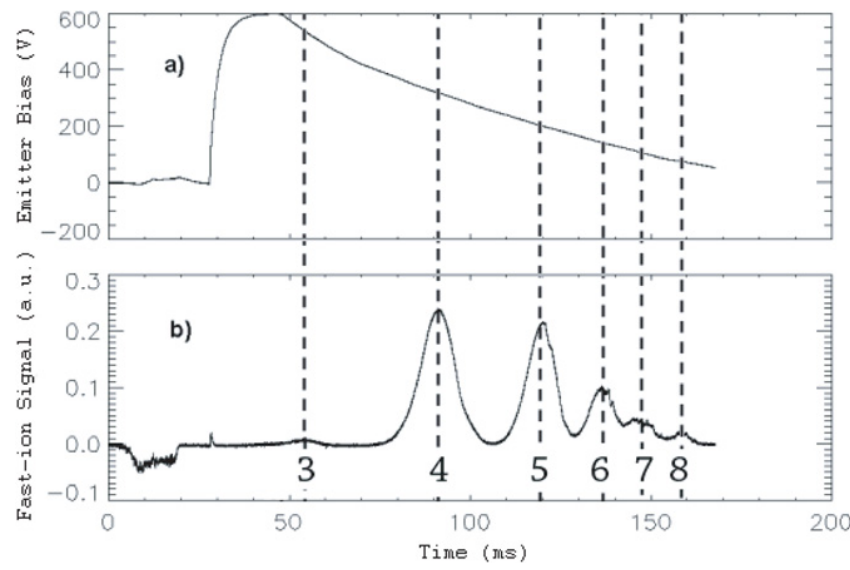

Figure 5. Finding the beam in the afterglow in an LAPD plasma. (a) Bias on the emitter and (b) fast-ion collector signal versus time. The labels by the peaks are the inferred number of cyclotron periods.

The shifts in beam position have two main origins. Changes in reference potential can alter the energy of the emitted beam; this effect was quite important for the argon RF source [12]. Another effect is a transverse jump of the beam as it emerges from the source. Because the source is perturbative, the electric potential on field lines that contact the source generally differs from the potential on open field lines. This difference produces a static electric field at the source that gives a radial 'kick' to the beam as it emerges from the source. Due to this effect, the beam position routinely jumps a few centimeters between the active and the afterglow phase in the LAPD (figure 6). More recently, experiments with a biased annulus provided a controlled, systematic study of the effect of dc electric fields [17]. Excellent agreement between measured and calculated orbital shifts is observed.

Some LAPD experiments employ magnetic mirror configurations. Empirically, the nominal position and pitch angle of

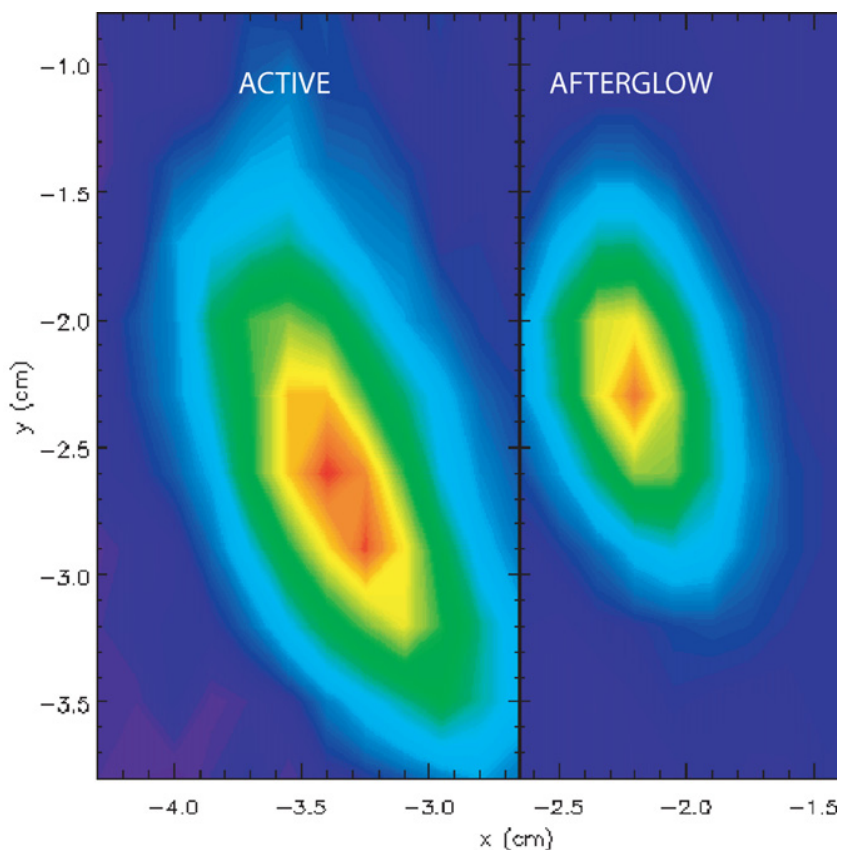

Figure 6. Motion of the beam profile between the active-phase and the afterglow of an LAPD plasma. The $400 \mathrm{eV}$ beam is $z=1.3 \mathrm{~m}$ from the source.

the beam is well described by the assumption that the magnetic moment $\mu=v_{\perp}^{2} / B$ is conserved, even when the conditions for $\mu$ conservation are inapplicable because the field $B$ changes in distances that are comparable to orbital dimensions. A representative example appears in figure 12 of [12].

In TORPEX, the magnetic field consists of a toroidal field $\left(B_{\mathrm{t}} \simeq 75 \mathrm{mT}\right)$ with a small vertical magnetic field component $\left(B_{v} \simeq 2 \mathrm{mT}\right)$. The resulting open field lines are helical and terminate on the lower and upper walls of the vessel. With this magnetic geometry, the fast ion motion without plasma 

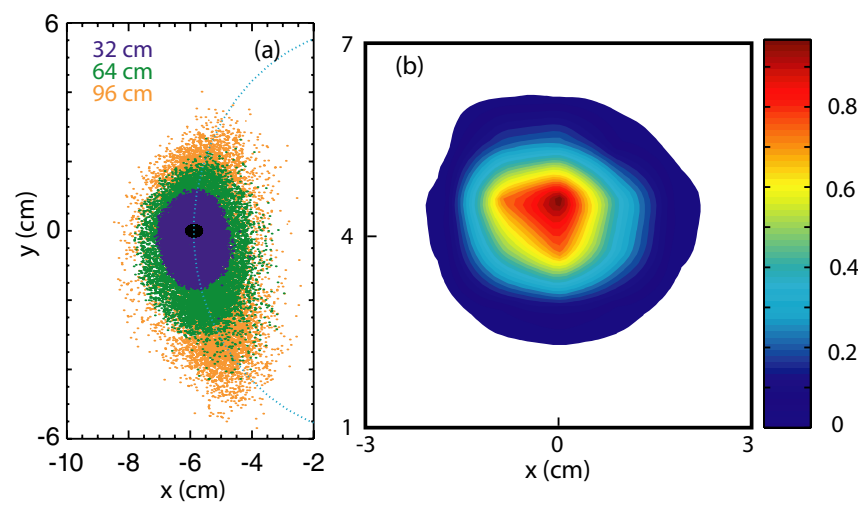

Figure 7. Simulations. (a) Radial and angular spreading caused by resonance with shear Alfvén waves in the LAPD. The colors represent particles collected at different $z$ planes. Reprinted with permission from [22]. Copyright 2008, American Institute of Physics. (b) TORPEX poloidal fast ion current density profile for ions of $70 \mathrm{eV}$ at a toroidal distance of $54 \mathrm{~cm}$ from the source reconstructed from the simulations with a synthetic diagnostic.

is a combination of the gyromotion along the field line and the vertical $\nabla B$ and curvature drifts. Thanks to the toroidal movable system of the source, a 3D beam profile can be reconstructed showing the contraction and expansion of the beam width, due to the gyromotion of the fast particles, and the broadening effect of the plasma on top of it $[11,18,19]$.

\subsection{Wave-field measurements}

In both devices, various probes and imaging cameras measure the wave properties. For an Alfvén wave study, the primary diagnostics are B-dot probes while, for electrostatic waves, sets of triple probes are often employed. All of the probes have widths smaller than the ion gyroradius. Tests with probes of different sizes show no measurable difference in wave properties. Measurements in TORPEX with a nonperturbative fast framing camera performed with and without electrostatic probes indicate that the small probes used for the fluctuation measurements do not appreciably alter the wave fields in terms of statistical and spectral properties of the electrostatic fluctuations. Also, insertion or removal of the TORPEX fast-ion source has a negligible effect on the turbulent waves.

In contrast, the LAPD fast-ion source is perturbative. One effect is modification of the electron density profile. The source blocks primary electrons emitted by the cathode, which reduces plasma production downstream of the source. To minimize this effect, fast ions inject upstream toward the cathode, where the density perturbation is smaller. In some cases (particularly for Alfvén waves), the presence of the source also alters the wave fields, so the waves are always measured with the source in place.

\subsection{Fast-ion simulations}

Simulation of the predicted beam spot (figure 7) is straightforward. Tracer ions emerge from the source with a specified position and velocity determined by the beam energy, divergence and aperture size. (In practice, the beam parameters in the simulation are adjusted to give good agreement to measurements acquired when Coulomb scattering and waveparticle interactions are negligible.) The vacuum magnetic field is specified, as well as any electromagnetic fields associated with waves. The Lorentz force law then determines the ion orbits. Since the particle orbits are computed by solving Newton's law with the Lorentz force, gyroaveraging is included implicitly to high accuracy. In LAPD simulations, Coulomb collisions periodically decrement the fast-ion energy and scatter the pitch angle. In both devices, the fluctuating fields throughout the volume are determined by theoretical modeling that is consistent with the measured fluctuations. For example, in TORPEX, the tracer particle trajectories are integrated in a turbulent electrostatic field resulting from the ideal interchange driven turbulence that is calculated by $2 \mathrm{D}$ simulations of the drift reduced Braginskii fluid equations $[20,21]$. Once the particles reach the collector, a synthetic diagnostic that mimics the detector computes $2 \mathrm{D}$ or $3 \mathrm{D}[18,19]$ profiles of the fast-ion current density for comparison with the data.

\section{Results to date}

\section{1. $L A P D$}

The first published physics results were a study of classical scattering [13]. The experiment was performed in the LAPD afterglow plasma with the argon fast-ion source and a biased gridded analyzer. The temporal decay in plasma density provided steadily decreasing Coulomb scattering rates. The measured energy deceleration (figure $8(a)$ ) and the radial spreading of the beam spot (figure $8(b)$ ) agree well with the predicted slowing-down and pitch-angle scattering rates. The attenuation of the beam due to charge exchange is also consistent with theoretical predictions. More recently, in studies focused on other effects, the radial spreading of the lithium beam caused by pitch-angle scattering has been measured in the active phase of LAPD plasmas, since it constitutes the irreducible minimum for the spreading rate. For example, in figure 12 of [9], pitch-angle scattering is responsible for the gradual spreading of the beam for the points labeled 'beam in plasma with no wave' and 'beam in afterglow plasma.' Like the earlier results, the recent results agree well with the classical transport equations summarized in [13].

All subsequent experiments used the lithium sources. The first studies of wave interactions with fast ions chose a simple case: the fast ions orbited in a single, coherent, shear Alfvén wave. The first experiment used linearly polarized shear Alfvén waves [22], while a subsequent experiment used circularly polarized waves [23]. In both cases, the Doppler-shifted cyclotron resonance, $\omega-k_{z} v_{z}=\Omega_{\mathrm{f}}$, was operative. (Here, $\omega$ and $k_{z}$ are the wave frequency and axial wave number, respectively.) The primary experiment was a scan of wave frequency to verify the resonance condition; at resonance, large radial spreading of the beam is observed (figure 9). In addition to frequency, the dependence on wave 

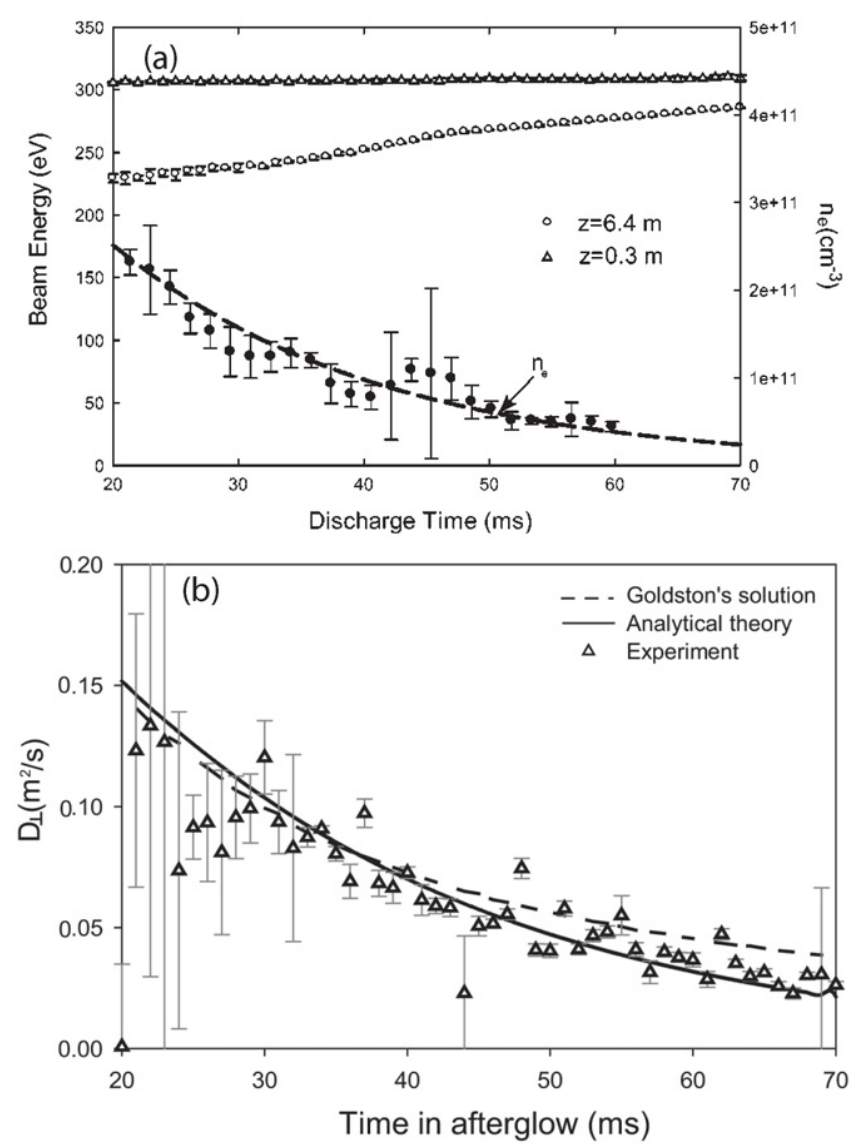

Figure 8. Slowing-down and pitch-angle scattering of an argon beam in the afterglow of an LAPD plasma. (a) Electron density and argon beam energy versus time. (b) Perpendicular diffusion coefficient inferred from radial spreading versus time. Two theoretical predictions are also shown. Reprinted with permission from [13]. Copyright 2005, American Institute of Physics.

amplitude [23], polarization [23], harmonic content [22] and perpendicular eigenfunction [22] was measured. All of the results are in good qualitative and quantitative agreement with simulations based on the measured properties of the shear Alfvén waves.

The most recent LAPD experiments have focused on fastion transport by turbulent wave fields. Obstacles placed in the plasma create a sharp density gradient that drives coherent and turbulent instabilities. Biasing the obstacle alters the plasma turbulence and, at large bias, generates new instabilities [24]. The first published experiment [25] studied the dependence of the transport on fast-ion gyroradius (figure 10(a)). Fast ions with varying gyroradii but the same $v_{z}$ orbited through the (primarily) electrostatic turbulence produced at the edge of a rectangular obstacle. As expected, the measured radial spreading of the beam is much greater for fast ions with small $\rho_{\mathrm{f}}$. Large gyroradius fast ions phase-average over the turbulence and are weakly affected by it. The effective potential experienced by the energetic ions is reduced by $J_{0}\left(k_{\perp} \rho_{\mathrm{f}}\right)$, so high energy ions experience less transport. (Here, $J_{0}$ is a Bessel function and $k_{\perp}$ is the perpendicular wavenumber of the dominant fluctuations.) Consistent with probe measurements, the fluctuations are modeled as twodimensional, electrostatic fields. In simulations, the ions are launched in various phases of the turbulent wave fields and a time-averaged spatial profile is assembled. The predicted radial spreading agrees well with experiment.

The second LAPD turbulence experiment [9] complements the first. In this experiment, the unperturbed fast-ion orbit is held fixed but the turbulence is varied. The fast ions execute helical orbits that are slightly smaller than the inner diameter of a biased, annular obstacle. The turbulence is altered by varying the plasma species and the bias on the obstacle. Figure 10(b) shows typical results for the measured spreading. Because the efficacy of orbit averaging is reduced at smaller $k_{\perp}$, the transport is largest in waves with larger spatial scales. Also, for waves of similar spatial scale, turbulent waves with shorter correlation lengths cause larger transport than coherent waves. All of the data are in good qualitative and quantitative agreement with theoretical expectations.

Another effect that was studied in the turbulence experiments is the time dependence of beam spreading. In general, the width of the beam spot $W$ as a function of time $t$ can be characterized by a coefficient $\gamma, W^{2} \propto t^{\gamma}$. If $\gamma=1$, the transport is diffusive; if $\gamma$ is larger (smaller) than one, the transport is called superdiffusive (subdiffusive). Experimentally, Coulomb collisions cause diffusive transport $(\gamma=1)$ for all distances from the source [25]. In contrast, at short distances from the source, the drift waves cause superdiffusive transport $(\gamma=2)$ [25]. This result is easily understood: for times short compared with a wave period, the electrostatic waves are virtually stationary, resulting in essentially ballistic radial transport of each ion. On the other hand, when the beam travels through the waves for half of a wave period, the electric field reverses sign and the direction of transport reverses, causing the beam to spread more gradually $(\gamma<1)$ [9]. At still greater distances from the source, the spreading approaches a diffusive regime [9].

\subsection{TORPEX}

A comprehensive theoretical investigation of the behavior of the fast ions as tracer particles in TORPEX plasmas was recently completed $[18,19]$. The study was performed in plasmas dominated by ideal interchange turbulence [21]. The results show that the dispersion of fast ions in the plane perpendicular to the magnetic field is generally nondiffusive, with $\sigma_{R}^{2}(t) \sim t^{\gamma}$, where $\gamma \neq 1$. Here, $\sigma_{R}^{2}(t)=\left\langle(R-\langle R\rangle)^{2}\right\rangle$ is the variance of the ion's radial positions. A large number of different ensembles of fast ions was used to explore the variations of the value of $\gamma$ as a function of two dimensionless quantities: the fast ion energy compared with the electron temperature, $\mathcal{E} / T_{\mathrm{e}}$, and the amplitude of turbulent fluctuations relative to the electron temperature. It was seen that, depending on the value of these parameters, the value of $\gamma$ can vary from $\gamma>1$ (superdiffusive) to $\gamma<1$ (subdiffusive) due to the effects of gyroaveraging and, more significantly, curvaturedrift averaging.

In TORPEX plasmas, using the vertical field intensity as a control parameter, the transition between an ideal interchange regime and a resistive interchange regime can be obtained. For high values of $B_{v}$, the first regime is obtained in which 

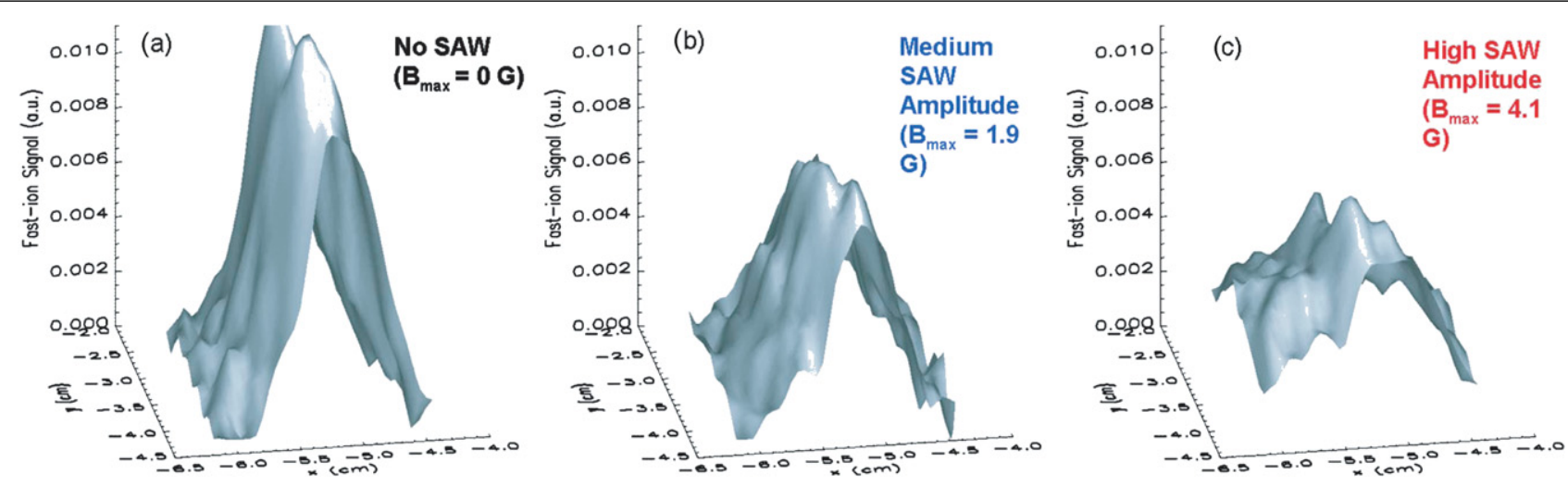

Figure 9. LAPD fast-ion beam profile with different shear Alfvén wave (SAW) amplitudes for lithium ions that are in cyclotron resonance with the wave. The $z$-axis is the fast-ion signal strength. (a) SAW antenna off. (b) Medium SAW amplitude (c) High SAW amplitude. Reprinted with permission from [23]. Copyright 2009, American Institute of Physics.
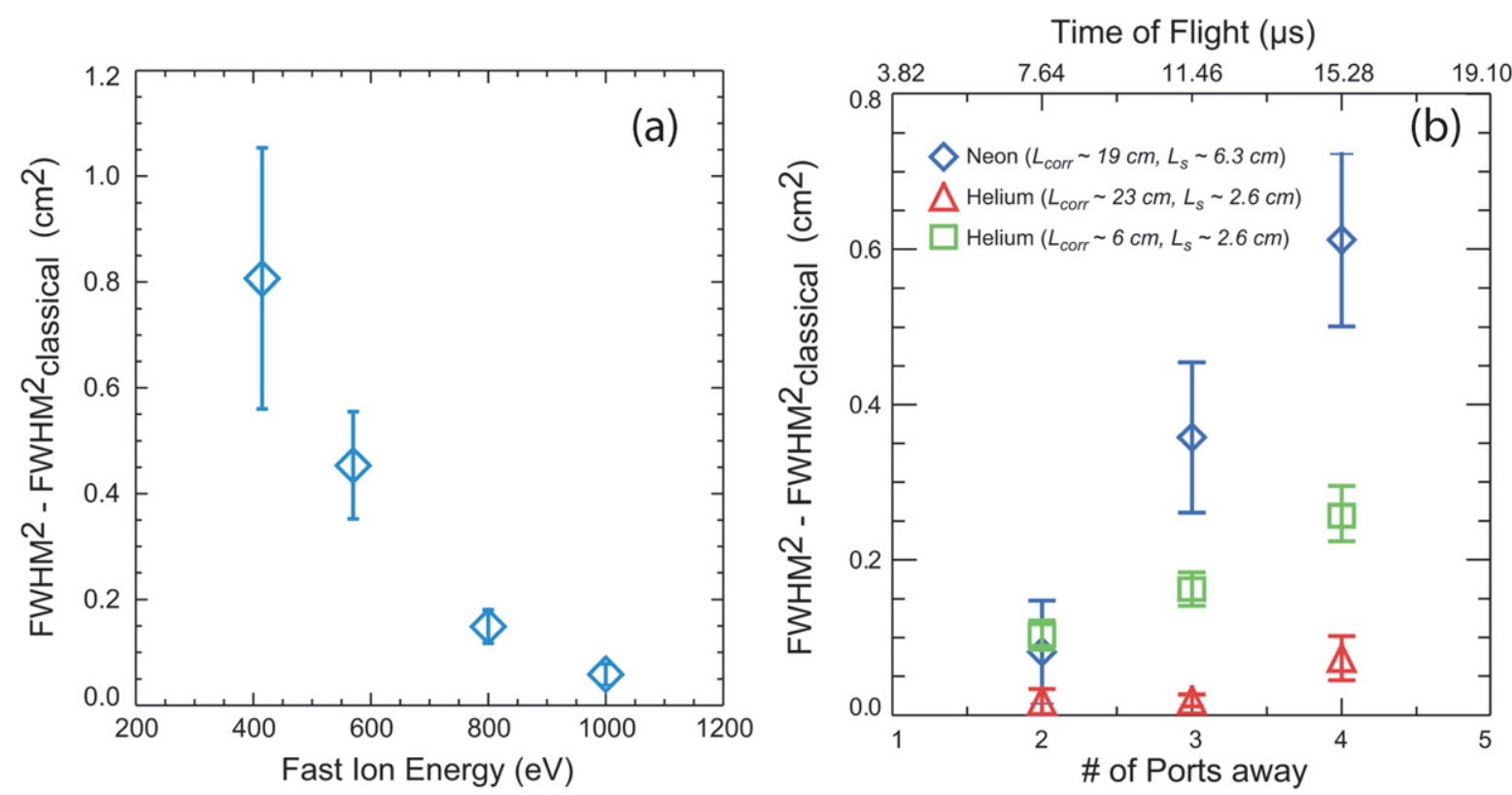

Figure 10. Drift-wave induced broadening of the beam width versus $(a)$ fast-ion energy and $(b)$ for different types of waves in the LAPD. Phase-averaging reduces the transport for large gyroradii, small scale size $L_{s}$ and long correlation length $L_{\text {corr }}$. Reprinted with permission from [17]. Copyright 2012, American Institute of Physics.

modes are observed with zero parallel wave numbers, $k_{\|}=0$, and perpendicular wave numbers, $k_{\perp}=2 \pi / \Delta$, where $\Delta=$ $2 \pi R_{0} B_{v} / B_{\mathrm{t}}$ is the vertical magnetic field line return distance, while, in the resistive interchange regime at lower $B_{z}$, finite $k_{\|}$ modes dominate the plasma dynamics [21, 26-28].

A first set of experiments with the toroidally moving system for the source was recently conducted and preliminary results are presented here. The experiments were conducted in a scenario with $B_{\mathrm{t}}=74 \mathrm{mT}$ and $B_{v}=2 \mathrm{mT}$, resulting in a simple magnetized torus (SMT) configuration with $\Delta \simeq$ $17 \mathrm{~cm}$, where the plasma is dominated by an ideal interchange mode, localized around the position of maximum pressure gradient, with wavenumbers of $k_{\|} \simeq 0$ and $k_{\perp} \simeq 37 \mathrm{~m}^{-1}$. The electron density at the injection location was $\sim 5 \times$ $10^{15} \mathrm{~m}^{-3}$ and the standard deviation of the floating potential time series, indicating the level of fluctuations, was $\sim 1 \mathrm{~V}$. These plasmas, similar to those extensively studied in [29-33] using electrostatic probes, contain a region on the low-field side where blobs are observed to propagate radially outward, resulting in intermittent transport of particles, heat, momentum and current [34-36].

Fast ions are injected in the blob region of TORPEX with an energy of $\sim 70 \mathrm{eV}$ and a horizontal orientation. The source is moved toroidally between each discharge over a total distance of $\sim 50 \mathrm{~cm}$ and poloidal profiles of the fast ion current are reconstructed at each position. Figure 4(b) shows an example of a fast ion current density profile at a toroidal distance of $\sim 54 \mathrm{~cm}$ from the source. The red cross indicates the position of the injection showing the displacement of the beam spot due to the vertical drift. Measurements are made with and without plasma, but with magnetic fields. The radial and vertical variances of the current profiles as a function of the toroidal angle $\phi$ are shown in figure 11 .

Simulations are run with source parameters based on measurements done without magnetic field. Ten thousand particles are launched with initial parameters modeled with 

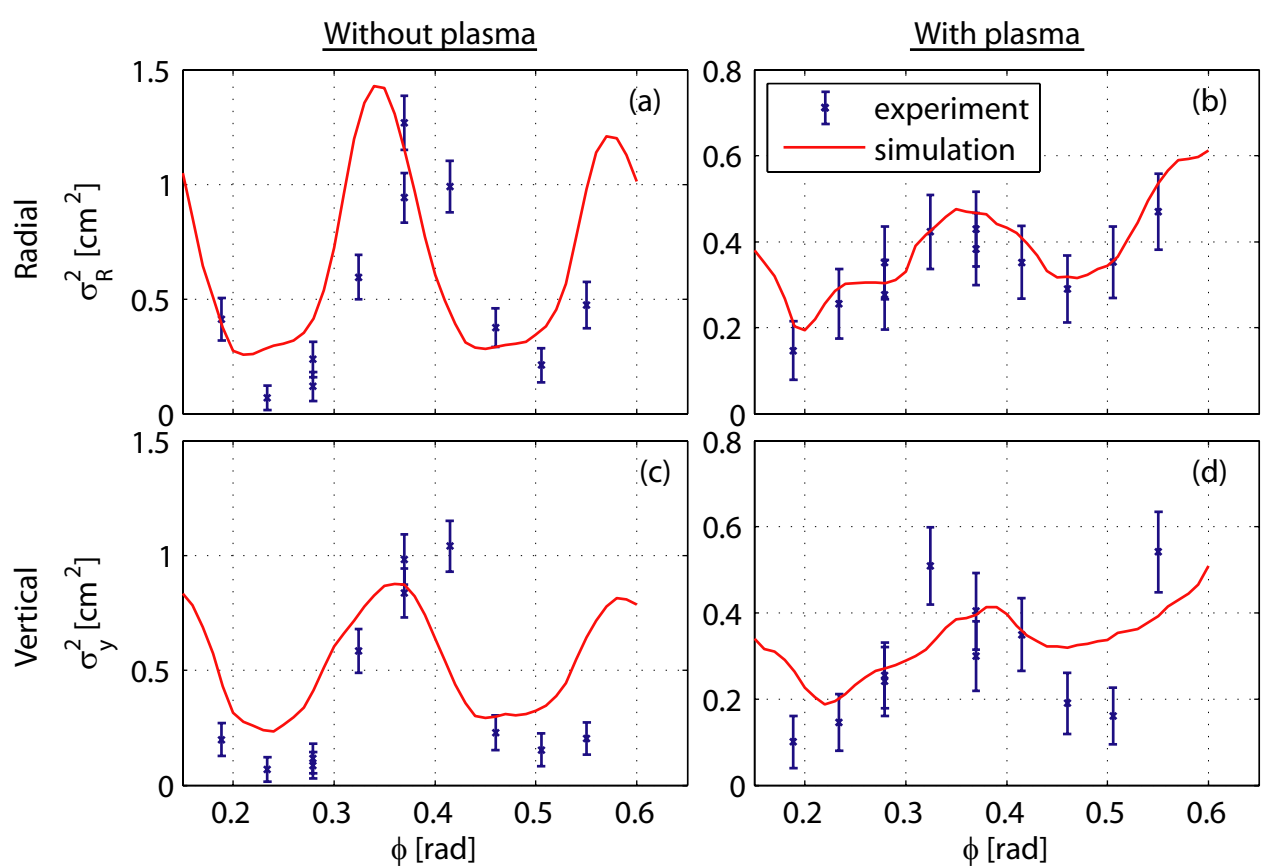

Figure 11. Radial (top) and vertical (bottom) fast ion beam width versus toroidal angle for ions of $70 \mathrm{eV}$ in TORPEX. The case without plasma (left) shows the oscillation of the beam width due to the Larmor motion of the particles. The case with plasma (right) shows the broadening effect of the plasma on top of the oscillation. Experiments and simulations show good agreement.

Gaussian distributions. The energy is $70 \mathrm{eV}$ with a standard deviation of $10 \%$. The initial position is $X_{0}=-0.4 \mathrm{~cm}$ and $Y_{0}=1.1 \mathrm{~cm}$ with standard deviation of $1.2 \mathrm{~mm}$. The initial values of the vertical and horizontal angles are $\alpha_{0}=-0.04 \mathrm{rad}$ and $\beta_{0}=0.04 \mathrm{rad}$, respectively, with standard deviation of $0.19 \mathrm{rad}$ and $0.13 \mathrm{rad}$, respectively. Simulations of the turbulence are performed with different values of the particle and heat sources in order to match experimental profiles. At the injection point, the fluctuation level of the floating potential is higher in the simulation than in the experiment. In order to match the potential fluctuations, the simulated plasma potential fluctuations, $\tilde{\phi}=\phi-\bar{\phi}$, are multiplied by a factor $\Xi=0.3$. Figure $7(b)$ shows an example of a fast ion current profile reconstructed with the synthetic diagnostic from the simulated fast ion trajectories. Figure 11 displays along the toroidal direction the variance of the beam profiles obtained with the synthetic diagnostic, from simulations, and from experimental measurements, which show similar features. For the case without plasma (figure 11(left)), this also provides a benchmark for the particle tracer solver. The oscillations of the variance of the beam due to the Larmor motion of the particles are clearly evident, although a small mismatch in the phase of the oscillations between experiment and simulation is observed. This is not observed in the presence of plasma, although the absolute value of the variance is decreased. Indeed, the spreading on the initial angle, in the simulations, had to be decreased by half in order to match the experiment. Those differences could be explained by the fact that the functioning of the source is affected by the surrounding plasma. In figure 11, the turbulent broadening of the beam is clearly revealed by the radial variance of the beam which increases as a function of the distance from the source. Numerical simulations at later times indicate that, under these conditions, fast ions undergo a subdiffusive transport with $\gamma \simeq 0.78$.

\section{Outlook}

To date, most experiments on the LAPD have relied on timeaveraged measurements of the radial spreading of the beam. In future work, it is desirable to develop techniques to measure wave-induced changes in energy and parallel velocity. A high bandwidth detector that can measure coherent fluctuations in beam current at wave frequencies is also desirable.

At TORPEX, the experimental setup is ready to explore the different fast ion transport regimes that are predicted by theory $[18,19]$ under different experimental conditions. For example, the ratio of fast ion energy to electron temperature can easily be changed. By injecting at different locations, in the mode or in the blob region, the turbulent fluctuations amplitude can be varied. Both gyro-averaging and drift-orbit averaging can be studied.

The development of the techniques described in section 2 and the subsequent successes (section 3) establish the groundwork for many future physics studies. At the LAPD, an obvious extension of the studies of fast-ion interaction with a single coherent shear Alfvén wave is to study the interaction with multiple shear Alfvén waves, particularly in a nonlinear regime. Another obvious opportunity is to measure the interaction of fast ions with compressional Alfvén waves at cyclotron harmonics.

At TORPEX, a toroidal current carrying wire will soon be installed in the middle of the chamber. This will allow us to study the fast ion transport in different magnetic configurations, from closed field lines to open field lines with rotational transform. 


\section{Acknowledgments}

The LAPD experiments were supported by DOE and conducted at the UCLA BaPSF user facility supported by the NSF/DOE. The authors gratefully acknowledge the invaluable assistance of the LAPD technical team.

The TORPEX experiments were supported in part by the Swiss National Science Foundation. The authors wish to acknowledge helpful discussions with Fabio Avino and Joaquim Loizu and acknowledge the inestimable support of the CRPP technical team.

\section{References}

[1] Breneman H H and Stone E C 1985 Astrophys. J. 229 L57

[2] Cattell C et al 1998 Geophys. Res. Lett. 252053

[3] Heidbrink W W and Sadler G J 1994 Nucl. Fusion 34535 and references therein

[4] Gekelman W et al 1991 Rev. Sci. Instrum. 622875

[5] Fasoli A et al 2006 Phys. Plasma 13055902

[6] Tripathi S K P, Pribyl P and Gekelman W 2011 Rev. Sci. Instrum. 82093501

[7] Zhang Y et al 2007 Rev. Sci. Instrum. 78013302

[8] Zhang Y 2008 Fast ions and shear Alfvén waves PhD Thesis University of California, Irvine http://www.physics.uci.edu/ $\sim$ wwheidbr/papers/Yang_thesis.pdf

[9] Zhou S et al 2011 Phys. Plasma 18082104

[10] Plyushchev G et al 2006 Rev. Sci. Instrum. 77 10F503

[11] Plyushchev G 2009 Interaction of supra-thermal ions with turbulence in a magnetized toroidal plasma $P h D$ Thesis EPFL, Lausanne

[12] Boehmer H et al 2004 Rev. Sci. Instrum. 751013
[13] Zhao L et al 2005 Phys. Plasma 12052108

[14] Zhao L 2005 Measurements of classical transport of fast ions PhD Thesis University of California, Irvine

[15] Zhou S 2011 Turbulent transport of energetic ions and thermal plasma PhD Thesis University of California, Irvine http:// www.physics.uci.edu/ wwheidbr/papers/Shu_thesis.pdf

[16] Bovet A, Furno I, Fasoli A, Gustafson K and Ricci P 2012 Nuclear Fusion 52094017

[17] Zhou S et al 2012 Phys. Plasma 19055904

[18] Gustafson K, Ricci P, Furno I and Fasoli A 2012 Phys. Rev. Lett. 108035006

[19] Gustafson K, Ricci P, Bovet A, Furno I and Fasoli A 2012 Phys. Plasmas 19062306

[20] Ricci P, Rogers B N and Brunner S 2008 Phys. Rev. Lett. 100225002

[21] Ricci P and Rogers B N 2010 Phys. Rev. Lett. 104145001

[22] Zhang Y et al 2008 Phys. Plasma 15102112

[23] Zhang Y et al 2009 Phys. Plasma 16055706

[24] Zhou S et al 2012 Phys. Plasma 19012116

[25] Zhou S et al 2010 Phys. Plasma 17092103

[26] Ricci P et al 2011 Phys. Plasmas 18032109

[27] Poli F M et al 2006 Phys. Plasmas 13102104

[28] Poli F M, Ricci P, Fasoli A and Podesta M 2008 Phys. Plasmas 15032104

[29] Furno I et al 2008 Phys. Rev. Lett. 100055004

[30] Furno I et al 2008 Phys. Plasmas 15055903

[31] Muller S H et al 2007 Phys. Plasmas 14110704

[32] Theiler C et al 2009 Phys. Rev. Lett. 103065001

[33] Diallo A et al 2008 Phys. Rev. Lett. 101115005

[34] Podestà M et al 2008 Phys. Rev. Lett. 101045001

[35] Labit B, Theiler C, Fasoli A, Furno I and Ricci P 2011 Phys. Plasmas 18032308

[36] Furno I et al 2011 Phys. Rev. Lett. 106245001 